\title{
Physiological roles of sigma factor SigD in Corynebacterium glutamicum
}

Hironori Taniguchi ${ }^{1,2}$, Tobias Busche ${ }^{2}$, Thomas Patschkowski ${ }^{2,3}$, Karsten Niehaus ${ }^{2,3}$, Miroslav Pátek ${ }^{4}$, Jörn Kalinowski ${ }^{2}$ and Volker F. Wendisch ${ }^{1,2^{*}}$ (i)

\begin{abstract}
Background: Sigma factors are one of the components of RNA polymerase holoenzymes, and an essential factor of transcription initiation in bacteria. Corynebacterium glutamicum possesses seven genes coding for sigma factors, most of which have been studied to some detail; however, the role of SigD in transcriptional regulation in C. glutamicum has been mostly unknown.

Results: In this work, pleiotropic effects of sigD overexpression at the level of phenotype, transcripts, proteins and metabolites were investigated. Overexpression of sigD decreased the growth rate of C. glutamicum cultures, and induced several physiological effects such as reduced culture foaming, turbid supernatant and cell aggregation. Upon overexpression of sigD, the level of Cmt1 (corynomycolyl transferase) in the supernatant was notably enhanced, and carbohydrate-containing compounds were excreted to the supernatant. The real-time PCR analysis revealed that sigD overexpression increased the expression of genes related to corynomycolic acid synthesis (fadD2, pks), genes encoding corynomycolyl transferases (cop 1, cmt1, cmt2, cmt3), L, D-transpeptidase (IppS), a subunit of the major cell wall channel $(p o r H)$, and the envelope lipid regulation factor (elrF). Furthermore, overexpression of sigD resulted in trehalose dicorynomycolate accumulation in the cell envelope.
\end{abstract}

Conclusions: This study demonstrated that SigD regulates the synthesis of corynomycolate and related compounds, and expanded the knowledge of regulatory functions of sigma factors in C. glutamicum.

Keywords: Corynebacterium glutamicum, Sigma factor, SigD, Mycomembrane, Trehalose dicorynomycolate

\section{Background}

Sigma factors are a component of bacterial RNA polymerase holoenzymes essential for promoter recognition and transcription initiation [1]. Most bacteria encode multiple sigma factors, and each sigma factor containing RNA polymerase holoenzyme initiates transcription from the cognate promoter sequences [2-5]. By replacing a sigma factor in RNA polymerase holoenzyme, bacteria activate transcription of a different gene set under different conditions, and cope with environmental changes [6]. Therefore, sigma factors play an important role in transcriptional regulation in a global manner. The knowledge of regulations by sigma factors is helpful to elucidate the regulatory network of the organism.

\footnotetext{
* Correspondence: volker.wendisch@uni-bielefeld.de

${ }^{1}$ Genetics of Prokaryotes, Faculty of Biology, Bielefeld University, Bielefeld, Germany

${ }^{2}$ Center for Biotechnology, Bielefeld University, Bielefeld, Germany Full list of author information is available at the end of the article
}

Corynebacterium glutamicum was first isolated as an organism secreting high amounts of L-glutamate [7]. Nowadays, this bacterium is used for production of Lamino acids in million tons per year, especially Lglutamate and L-lysine [8]. C. glutamicum ATCC 13032 has seven sigma factor genes in its chromosome, sigA, $\operatorname{sig} B, \operatorname{sig} C$, sigD, sigE, sigH and $\operatorname{sig} M[9,10]$. The physiological functions of SigA, SigB, SigC, SigE, SigH and SigM have been studied to some extent [11]; however, the regulation and physiological roles of SigD in C. glutamicum have not yet been revealed. sigD gene is well conserved among corynebacteria, and 17 out of 19 examined Corynebacterium species possess sigD genes [11]. Therefore, it is assumed that SigD plays a substantial role in transcriptional regulation and subsequent adaptation of C. glutamicum to changing environments.

C. glutamicum belongs to the CMN (Corynebacterium, Mycobacterium, Nocardia) group, which is characterized by the unique molecular constituents of their cell 
envelopes such as the mycomembrane [12]. The mycomembrane is composed of a monolayer of corynomycolate ( $\alpha$-alkyl, $\beta$-hydroxy fatty acid), which is covalently linked to arabinogalactan or forms other lipids such as trehalose monocorynomycolate (TMCM) and trehalose dicorynomycolate (TDCM) [13].

In this work, we evaluated the effects of deletion and overexpression of $\operatorname{sig} D$ on the cell phenotype, and revealed the influence of sigD overexpression on transcripts, proteins and metabolites. The achieved results disclosed the important roles of SigD in mycomembrane synthesis and maintaining cell wall integrity.

\section{Methods}

\section{Bacterial strains, plasmids and oligonucleotides}

The strains, plasmids and oligonucleotides used in this work are listed in Additional file 1: Table S1. A plasmid for sigD overexpression was constructed based on pVWEx1, which is an IPTG inducible expression vector for E. coli and C. glutamicum [14]. A plasmid for gene disruption was constructed based on pK18mobsacB [15]. For plasmid construction, DNA fragments were amplified from the genomic DNA of C. glutamicum ATCC 13032 by PCR with the oligonucleotide pairs shown in Additional file 1: Table S1. These fragments were inserted into the digested plasmid by ligation or Gibson assembly $[16,17]$. E. coli DH5 $\alpha$ was used for cloning. $E$. coli competent cells were transformed by the heat shock method [16] or by the electroporation method [18]. DNA sequences of all cloned DNA fragments were confirmed to be correct by sequencing. C. glutamicum competent cells were transformed by electroporation at $2.5 \mathrm{kV}, 200 \Omega$, and $25 \mu \mathrm{F}[8,19]$. Gene disruption via two-step homologous recombination and the following selections were carried out as previously described [8]. Disruption was verified by PCR with the respective oligonucleotide pairs.

\section{Medium and conditions for growth experiments}

Unless otherwise specified, C. glutamicum cells were precultured overnight in lysogeny broth (LB) medium [16] supplemented with $56 \mathrm{mM}$ of glucose, washed once with chemically defined CGXII medium [8] without carbon source, and then inoculated into CGXII medium with $222 \mathrm{mM}$ of glucose at an initial $\mathrm{OD}_{600}$ of 1 . The cultivation was performed at $30^{\circ} \mathrm{C}, 120 \mathrm{rpm}$. $\mathrm{OD}_{600}$ was measured with UV-1202 spectrophotometer (Shimadzu, Duisburg, Germany) with suitable dilutions. When necessary, $25 \mu \mathrm{g} / \mathrm{mL}$ of kanamycin and appropriate concentrations of IPTG were added as indicated in the text. For the growth experiment in BioLector ${ }^{\circledR}$ cultivation system (m2pLabs, Baesweiler, Germany), cells were cultivated in $1 \mathrm{~mL}$ of CGXII medium with $222 \mathrm{mM}$ of glucose using FlowerPlate $^{\bullet}(\mathrm{m} 2 \mathrm{pLabs}$, Baesweiler,
Germany) at $30{ }^{\circ} \mathrm{C}, 1100 \mathrm{rpm}$. Cell growth was monitored online every $10 \mathrm{~min}$, and the maximum growth rate $\left(h^{-1}\right)$ was determined from the growth rates $\mu\left(h^{-1}\right)$ which were calculated with regression analysis from backscattering light intensity (wavelength of $620 \mathrm{~nm}$ ) at 20 consecutive measuring points.

\section{Photometric determination of supernatant turbidity and observation of cell aggregation by microscopy}

To quantify turbidity of supernatants, cell cultures were centrifuged for $30 \mathrm{~min}$ with $15,000 \times \mathrm{x}$ at room temperature. The absorption of supernatants was measured at a wavelength of $600 \mathrm{~nm}$. For microscopic imaging, the culture of each strain in the stationary phase was diluted in CGXII medium without carbon source to an $\mathrm{OD}_{600}$ of 1 , and observed by microscopy with $100 \mathrm{x}$ oil immersion objective lens and $10 \mathrm{x}$ ocular lens. Quantification of cell aggregate size was performed using Image) (https://imagej.nih.gov/ij/).

\section{Protein analysis of the supernatant}

Supernatants were taken from the stationary phase cultures. Four volumes of acetone were added to one volume of supernatant, and stored at $-20^{\circ} \mathrm{C}$ overnight. After centrifugation at $4{ }^{\circ} \mathrm{C}, 20,000 \times \mathrm{g}$ for $15 \mathrm{~min}$, precipitates were resuspended in $20 \mathrm{mM}$ Tris- $\mathrm{HCl}$ (pH 7). SDS-PAGE was performed using Tris-glycine discontinuous buffer, and visualized by staining with Coomassie Brilliant Blue R250 as previously described [16]. Quantification of the intensity of each band was performed using ImageJ. Protein bands with different intensities observed in the control strain and the sigD overexpressing strain were excised from SDS-PAGE gels and transferred to a new tube which had been washed with trifluoroacetic acid: acetonitrile: $\mathrm{H}_{2} \mathrm{O}$ $(0.1: 60: 40 \mathrm{v} / \mathrm{v})$ in advance. The digestion of protein in the excised band was performed with trypsin overnight as previously described [20]. Protein sequences were identified using an ultrafleXtreme MALDI-TOF/TOF mass spectrometry (Bruker, Bremen, Germany) and Mascot search engine (Matrix Science, London, UK) as previously described with some modifications for C. glutamicum ATCC 13032 [21].

\section{Quantification of carbohydrate in acetone precipitates}

Supernatants were precipitated with acetone as described above. Precipitates were resuspended with $100 \mu \mathrm{L}$ of $20 \mathrm{mM}$ Tris- $\mathrm{HCl}$ ( $\mathrm{pH}$ 7), placed at room temperature for $30 \mathrm{~min}$, and then the insoluble fraction was recovered by centrifugation. Insoluble fractions were hydrolyzed as previously described [22]. Briefly, the precipitates were resuspended with $75 \mu \mathrm{L}$ of $72 \%(w / w)$ sulfuric acid and incubated at room temperature for $3 \mathrm{~h}$. The slurry was diluted to $1 \mathrm{~mL}$ with water, heated at $100{ }^{\circ} \mathrm{C}$ for $4 \mathrm{~h}$, and cooled down on ice. Colorimetric quantification for 
carbohydrates was performed by the phenol sulfuric acid method as previously described with some modifications $[23,24]$. Briefly, $200 \mu \mathrm{L}$ of hydrolysate was mixed with $600 \mu \mathrm{L}$ of concentrated sulfuric acid rapidly, and $120 \mu \mathrm{L}$ of $5 \%$ phenol $(w / v)$ in water was added immediately. The mixture was incubated for $5 \mathrm{~min}$ at $90{ }^{\circ} \mathrm{C}$, and cooled to room temperature for $5 \mathrm{~min}$. The absorption at $490 \mathrm{~nm}$ was measured, and compared to the absorption of the control samples with different concentrations of arabinose.

\section{RNA extraction}

Cells were first precultured in LB medium, and inoculated in CGXII medium with $222 \mathrm{mM}$ of glucose for adaptation. Then, the appropriate amount of cell culture was inoculated into fresh CGXII medium with $222 \mathrm{mM}$ of glucose at an initial $\mathrm{OD}_{600}$ of 1 . The cells were harvested at an $\mathrm{OD}_{600}$ between 6 and 8 . Cell cultures $(1 \mathrm{~mL})$ were centrifuged for $30 \mathrm{~s}$ at 20,000 $\mathrm{x} g$, and immediately frozen with liquid nitrogen after removing supernatant. RNA isolation was performed using the RNeasy mini kit along with the RNase-free DNase set (Qiagen, Hilden, Germany) as previously described [25]. The absence of contaminating genomic DNA in RNA samples was confirmed by PCR with multiple oligonucleotide pairs specific to genomic DNA.

\section{RNA isolation, library preparation and RNA-seq}

For transcriptome sequencing, RNA samples isolated individually from biological triplicates were mixed for each stain. Quality check of the isolated RNA, library preparation, RNA-seq and data analysis were performed as previously described [26]. Briefly, RNA quality was checked by Trinean Xpose (Gentbrugge, Belgium) and Agilent RNA Nano 6000 kit with Agilent 2100 Bioanalyzer (Agilent Technologies, Böblingen, Germany). Ribo-Zero rRNA Removal Kit (Bacteria) from Illumina (San Diego, CA, USA) was used to remove the ribosomal RNA molecules from the isolated total RNA. Removal of rRNA was checked by Agilent RNA Pico 6000 kit on Agilent 2100 Bioanalyzer (Agilent Technologies, Böblingen, Germany). TruSeq Stranded mRNA Library Prep Kit from Illumina (San Diego, CA, USA) was used to prepare cDNA libraries. cDNAs were sequenced paired end on an Illumina MiSeq system (San Diego, CA, USA) using 50 bp read length.

\section{Read mapping, data visualization and analysis of gene expression}

Trimmed reads (26 nt) were mapped to the C. glutamicum ATCC 13032 reference genome sequence [9] with SARUMAN [27], allowing for up to one error per read. The forward and reverse read, if both present and with a maximum distance of $1 \mathrm{~kb}$, were combined to one read that contains the reference sequence as insert. Paired mappings with a distance $>1 \mathrm{~kb}$ were discarded, and paired reads with either only the forward or only the reverse read mapping were retained as single mapping reads, as previously described [28]. ReadXplorer 2.2.0 was used for visualization of short read alignments [29]. For differential gene expression analysis, ReadXplorer and Bioconductor package DESeq implemented in ReadXplorer were used $[29,30]$. Genes with the mean value of signal intensity less than 30 were discarded. A-value and M-value of each gene were calculated based on the intensity value of the strain of interest and the control strain.

\section{Real-time PCR analysis}

For real-time PCR analysis, relative abundance of mRNA of each gene was quantified using the same amount of total RNA. RNA sample extracted from biological triplicates were quantified individually. The experiment and analysis were performed as previously described with respective oligonucleotide pairs in Additional file 1: Table S1 [31].

\section{Detection of trehalose dicorynomycolate by thin layer chromatography}

Lipid extraction and thin layer chromatography (TLC) were performed based on the method previously described [32, 33]. Briefly, the crude lipid was extracted from cell pellet with $\mathrm{CHCl}_{3} / \mathrm{CH}_{3} \mathrm{OH}(1: 1 \mathrm{v} / \mathrm{v})$ once, and with $\mathrm{CHCl}_{3} / \mathrm{CH}_{3} \mathrm{OH}(2: 1 \mathrm{v} / \mathrm{v})$ for three times. All the extracts were pooled for each strain, and mixed with $\mathrm{CHCl}_{3} / \mathrm{CH}_{3} \mathrm{OH} / \mathrm{H}_{2} \mathrm{O}(8: 4: 2 \mathrm{v} / \mathrm{v})$ resulting the aqueous layer and the organic layer. The lower organic layer was collected and evaporated to dryness. The dried lipid was weighed and resuspended with $\mathrm{CHCl}_{3} / \mathrm{CH}_{3} \mathrm{OH}$ $(4: 1 \mathrm{v} / \mathrm{v})$ to the same concentration for each sample. TLC was performed with ALUGRAM SIL G/UV254 (Macherey-Nagel, Germany) with $\mathrm{CHCl}_{3} / \mathrm{CH}_{3} \mathrm{OH} / \mathrm{H}_{2} \mathrm{O}$ $(30: 8: 1 \mathrm{v} / \mathrm{v})$ as a developing solvent. Total lipid $(600 \mu \mathrm{g})$ was developed for each strain, and the bands were visualized by spraying TLC plate with sulfuric acid and heating to $110{ }^{\circ} \mathrm{C}$. The mobility of TDCM or TMCM was determined by the retardation factor (Rf) value from the previous studies [32,33]. Quantification of the intensity of each band was performed with ImageJ.

\section{Results}

Deletion and overexpression of sigD influenced the maximum growth rate

The $\operatorname{sig} D$ deletion mutant $(\Delta \operatorname{sig} D)$ and the $\operatorname{sig} D$ overexpressing strain were cultivated in CGXII medium containing $222 \mathrm{mM}$ of glucose as carbon source. sigD was overexpressed from the plasmid pVWEx1-sigD using an IPTG inducible promoter and different IPTG concentrations $(0,10,50,250$ or $1000 \mu \mathrm{M})$. The $\Delta s i g D$ mutant grew slightly slower than the wild type (WT) strain (Fig. 1a). The maximum growth rate of the sigD overexpressing strain WT(pVWEx1-sigD) decreased in an IPTG- 

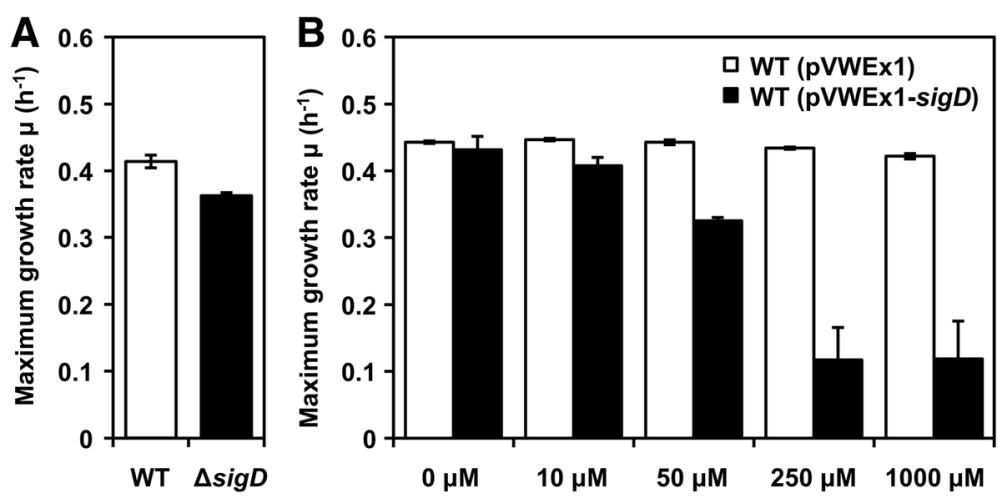

Fig. 1 Maximum growth rates of the $\Delta$ sigD strain and the sigD overexpressing strain with different IPTG concentrations. The maximum specific growth rate $\left(\mathrm{h}^{-1}\right)$ was shown for (a) C. glutamicum WT and $\Delta$ sigD, and (b) WT(pWWEx1) and WT(pWWEx1-sigD) with different IPTG concentrations $(0,10,50,250,1000 \mu \mathrm{M})$. Error bars represent standard deviations from biological triplicates

dependent manner, which was not observed for WT(pVWEx1) (Fig. 1b). Higher concentrations of IPTG (250 and $1000 \mu \mathrm{M}$ ) severely inhibited the growth of WT(pVWEx1-sigD). The final biomass concentrations after $24 \mathrm{~h}$ of cultivation were comparable for WT, $\Delta \operatorname{sig} D, \mathrm{WT}(\mathrm{pVWEx} 1)$ and WT(pVWEx1-sigD) with 0, 10 or $50 \mu \mathrm{M}$ of IPTG (data not shown). The slower growth of the $\Delta s i g D$ strain indicated that $\operatorname{sig} D$ is beneficial for growth in minimal CGXII medium although it is not essential for growth under optimum conditions. As excessive sigD overexpression at the high IPTG concentrations was found to be harmful to cells, induction with $50 \mu \mathrm{M}$ of IPTG was used for further experiments.

sigD overexpression influenced cell culture characteristics WT(pVWEx1-sigD) with $50 \mu \mathrm{M}$ of IPTG showed distinct characteristics compared to WT(pVWEx1) as the cultures of WT(pVWEx1-sigD) foamed significantly less than those of WT(pVWEx1) (Fig. 2a). Cell cultures with different IPTG concentrations $(0,10,50 \mu \mathrm{M})$ using FlowerPlates and a BioLector system revealed that the supernatants of WT(pVWEx1-sigD) cultures showed higher turbidity than those of the control strain, and this turbidity increased in an IPTG-dependent manner (Fig. 2b). In addition to these distinct characteristics of the cell culture, cell aggregation was observed under the microscope in WT(pVWEx1-sigD) with IPTG (Fig. 2c-e). Taken together, overexpression of sigD induced pleiotropic changes of the phenotype of C. glutamicum.

\section{sigD overexpression changed the pattern of secreted proteins}

Overexpression of $\operatorname{sig} D$ influenced the culture characteristic of foaming. Therefore, the profile of proteins in the culture supernatant was compared between WT(pVWEx1) and WT(pVWEx1-sigD). Proteins in the supernatants were analyzed by $1 D$ SDS-PAGE after concentrating by acetone precipitation. We observed different band patterns of proteins secreted by WT(pVWEx1) and WT(pVWEx1-sigD) (Fig. 3a). The proteins in the bands with different intensity were further characterized by tryptic digestion and MALDI-TOF/TOF mass spectrometry (Fig. 3b, c). In this way, corynomycolyl transferase Cmt1 was identified in the band 3, which showed a higher intensity when $\operatorname{sig} D$ was overexpressed. The band 2 was identified as L, D-transpeptidase LppS, and the band 4 was identified as mixture of two proteins, corynomycolyl transferase Cmt2 and putative secreted protein Cg2052. On the other hand, Psp3, which was found in the band 1 and annotated as putative secreted protein, was less abundant in WT(pVWEx1-sigD). These results demonstrated that overexpression of sigD altered the secreted protein profile. Since Cmt1 abundance in the supernatant increased most upon sigD overexpression (Fig. 3a, c), cmt1 was overexpressed in the WT strain. However, less foaming was not observed under these conditions (data not shown). Therefore, we concluded that less foaming of the culture did not occur only due to Cmt1 protein abundance but to complex or other unknown reasons.

\section{sigD overexpression induced the secretion of carbohydrate-containing compounds}

During acetone precipitation of the supernatant, we observed an insoluble fraction only in the sample of the sigD overexpressing strain. This insoluble fraction still existed after an overnight protease $\mathrm{K}$ treatment. For the strain C. glutamicum CGL2005, it was reported that the ethanol-precipitated fraction of extracellular components consisted primarily of carbohydrates [34]. Therefore, the carbohydrate content in this fraction was determined by the phenol sulfuric acid method [23], which detects all classes of carbohydrates [35]. Arabinose, which is one of the components of peptidoglycan in C. glutamicum, was used as control. Colorimetric determination after 


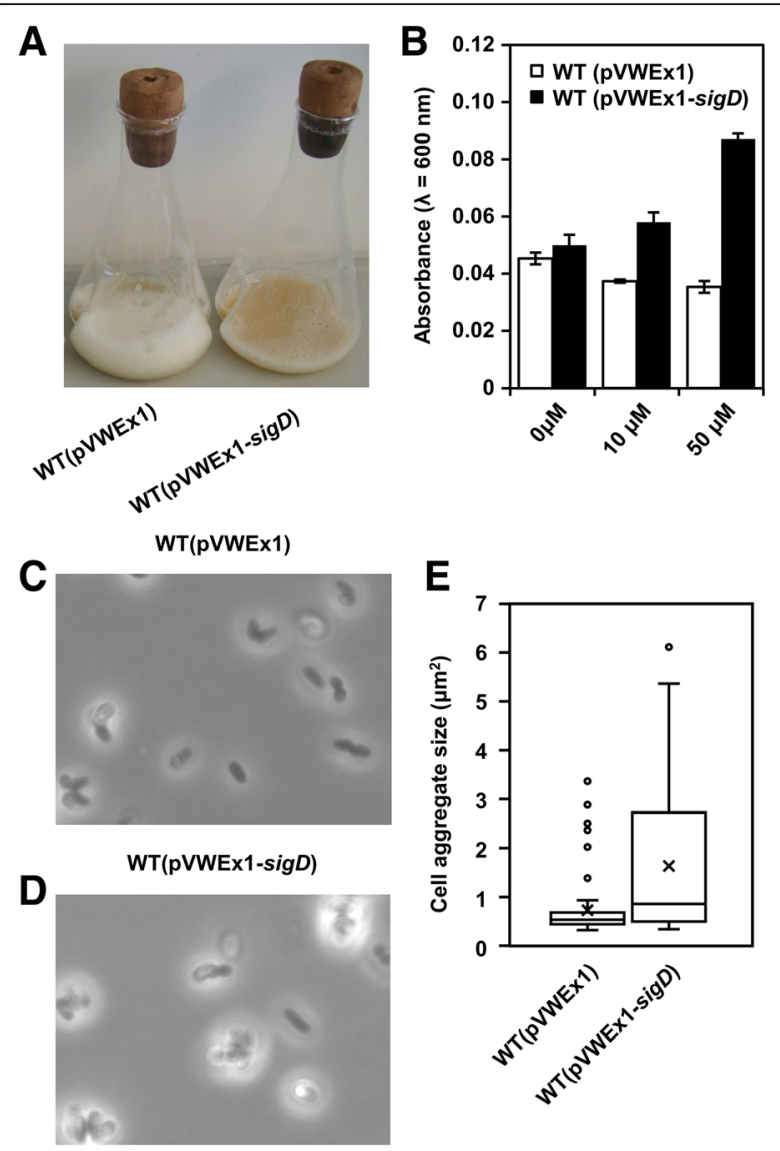

Fig. 2 Influence of sigD overexpression on cell cultures and cell morphology. a Cell cultures with $50 \mu \mathrm{M}$ of IPTG after $36 \mathrm{~h}$ of cultivation are shown. $\mathbf{b}$ Supernatant turbidity with different concentrations of IPTG $(0 \mu \mathrm{M}, 10 \mu \mathrm{M}, 50 \mu \mathrm{M})$ after $36 \mathrm{~h}$ is shown. Error bars represent standard deviations from biological triplicates. Microscopic images of the WT strain (c) and the sigD overexpressing strain (d) are shown. Cells in the stationary phase were observed under the microscope with a magnification of 1000. e Distribution of the size of cell aggregates is shown. The size of cell aggregates was analyzed by ImageJ, and the distribution was visualized by the boxand-whisker plot. Lower whisker, lower quantile, median, upper quantile and upper whisker are shown. The cross point indicates mean, and outliners were plotted as individual points

hydrolysis confirmed that the insoluble fraction of WT(pVWEx1-sigD) supernatants contained carbohydrates (arabinose equivalent of $1.3 \mathrm{mM}$ ). On the other hand, the same treatment of WT, $\Delta s i g D$ or WT(pVWEx1) supernatants resulted in carbohydrate contents below the detection limit (arabinose equivalent $<0.1 \mathrm{mM}$ ) (Table 1). These results showed that $\operatorname{sig} D$ overexpression induced the secretion of polysaccharides or carbohydrate-containing compounds into the supernatant.

\section{SigD regulated transcription of several genes related to cell envelope integrity}

RNA-seq and real-time PCR analysis were performed to understand the effects caused by $\operatorname{sig} D$ overexpression or deletion at the transcriptional level. The relative abundance of mRNA of each gene was compared in WT(pVWEx1-sigD) without IPTG or with $50 \mu \mathrm{M}$ of IPTG to analyze the effects of sigD overexpression. On the other hand, the mRNA abundance was compared between the $\triangle \operatorname{sig} D$ and the WT strain to study the effects of sigD deletion. First, RNA - seq analysis was applied to screen the genes whose expression levels changed upon sigD overexpression or deletion. RNA-seq analysis implied that expression of 29 genes increased upon $\operatorname{sig} D$ overexpression $(\mathrm{M}$-value $>1$ ) (Additional file 2: Table S2). Of these 29 genes, several genes are annotated as genes related to cell wall integrity; 6 genes (cop1, $c m t 1, c m t 2, c m t 3, e l r F$ and fadD2) as corynomycolyl related proteins, a gene annotated as L, D-transpeptidase (lppS) and three genes (cg0420, cg0532, cg1181) as glycosyltransferases. In addition, expression of two further genes encoding proteins with mycomembrane-related functions, porH (cg3009) and pks (cg3178), increased upon $\operatorname{sig} D$ overexpression (M-value $>\log _{2}(1.5)$ ) and slightly decreased in the $\Delta s i g D$ strain (M-value $<-\log _{2}(1.5)$ ). Based on these RNA-seq results, the expression of those genes was further analyzed by real-time PCR (Fig. 4). All of the tested genes were shown to be upregulated significantly as consequence of $\operatorname{sig} D$ overexpression. Increased transcript levels of cmt1, cmt2 and $\operatorname{lpp} S$ were consistent with the increased protein levels of Cmt1, Cmt2 and LppS in culture supernatants upon sigD overexpression (Fig. 3b, c). These results showed that the function of SigD is related to the regulation of cell envelope integrity such as mycomembrane synthesis and cell wall synthesis.

\section{Overexpression of $s i g D$ increased the amounts of trehalose dicorynomycolate}

The results of the real-time PCR analysis confirmed that the expression of several genes annotated as corynomycolyl transferase (cop1, cmt1, cmt2, cmt3) and genes related to corynomycolic acid production ( $p k s$ and $f a d D 2$ ) increased due to $\operatorname{sig} D$ overexpression. Overexpression of cop1 is known to increase the trehalose dicorynomycolate (TDCM) content in the cells [33]. To confirm the effect of $\operatorname{sig} D$ overexpression on the TDCM content, the crude lipid was extracted from cells, and its composition was analyzed by TLC (Fig. 5). The intensity of the band corresponding to TDCM increased 39\% upon sigD overexpression. On the contrary, the intensity of the band corresponding to trehalose monocorynomycolate (TMCM) did not show a notable difference between the two strains. This result indicates that upregulation of multiple genes by sigD overexpression increased the flux toward corynomycolic acid synthesis which resulted in an alteration of TMCM/ TDCM ratio as well as accumulation of other carbohydrate containing compounds. 

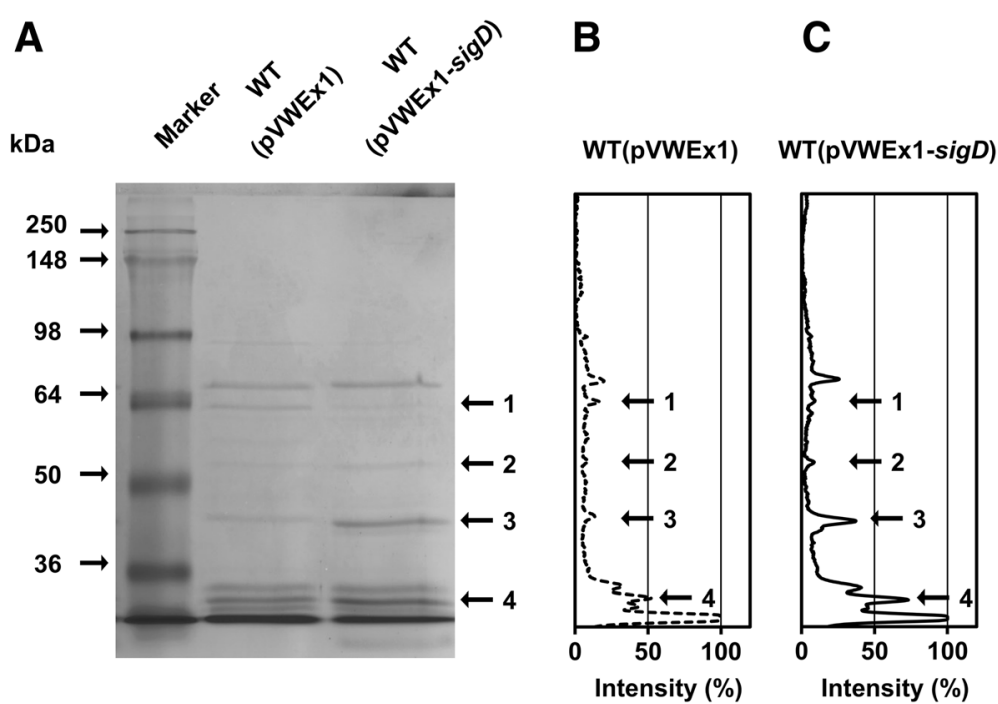

Fig. 3 1D-SDS PAGE of proteins in the supernatants. a Secreted proteins were analyzed by 12\% SDS-PAGE. The molecular sizes of proteins in the marker are shown in kDa. WT(pWWEx1) and WT(pVWEx1-sigD) protein samples were obtained by acetone precipitation of the supernatants. Proteins from $200 \mu \mathrm{L}$ of the supernatant was loaded on each lane. The intensity of secreted protein bands was quantified for WT(pWWEx 1) (b) and for WT(pWWEx1-sigD) (c). The highest intensity of the band was normalized to $100 \%$. The protein bands labeled with numbers were subjected to MALDI-TOF/TOF MS. 1: Psp3 (Cg2061), 2: LppS (Cg2720), 3: Cmt1 (Cg0413), 4: Cmt2 (Cg3186) and Cg2052

\section{Discussion}

Understanding the regulatory mechanisms of bacteria is important in many fields varying from biotechnology to public health. C. glutamicum has been used for the production of amino acids for several decades, however, the transcriptional regulation by sigma factors has not been fully elucidated. As for SigD, selection of high oxygen requiring mutants in a transposon library accidentally revealed that deletion of $\operatorname{sig} D$ in $C$. glutamicum loses the ability to grow under low oxygen concentrations [36], however, the knowledge is still limited. In the previous works, we demonstrated that overexpression of one of global regulators can artificially perturb the cellular regulation and influence metabolites as well as transcripts [37, 38]. This approach of sigma factor gene overexpression was found to be a useful approach for investigation of regulatory mechanisms and activation of specific biosynthesis pathways in C. glutamicum. In this work, deletion and overexpression of $\operatorname{sig} D$ revealed that sigma factor SigD plays a role in regulating maintenance of the cell wall integrity in C. glutamicum (Fig. 6).

Table 1 Carbohydrate content in acetone precipitated culture supernatants

\begin{tabular}{lllll}
\hline & WT & $\Delta$ sigD & WT(pWWEx1) & WT(pWWEx1-sigD) \\
\hline $\begin{array}{l}\text { Arabinose equivalent } \\
\text { carbohydrate (mM) }\end{array}$ & $<0.1$ & $<0.1$ & $<0.1$ & $1.29 \pm 0.28$ \\
\hline
\end{tabular}

Carbohydrate content was measured by the phenol sulfuric acid method. Arabinose samples with known concentrations were used as standards. Carbohydrate content was calculated to the concentration $(\mathrm{mM})$ of arabinose equivalent carbohydrate. The detection limit is $0.1 \mathrm{mM}$. Standard deviations were calculated from three biological replicates
The real time PCR analysis revealed that overexpression of $\operatorname{sig} D$ induced the expression of multiple genes related to mycomembrane synthesis (cop $1, c m t 1, c m t 2$, cmt3, elrF, fadD2, porH and pks) which exist at the different loci in the C. glutamicum genome. For mycomembrane biosynthesis, one molecule of fatty acid is carboxylated via the carboxylation complex composed of AccD2, AccD3, AccBC and AccE [39], and a second fatty acid is activated to a fatty acyl-CoA by FadD2 [40]. These two molecules are condensed and attached to trehalose by Pks $[41,42]$. This product is reduced to TMCM (trehalose monocorynomycolate) by CmrA [43], and TMCM is exported from cytoplasm [44, 45]. Then, corynomycolyl transferases transfer the corynomycolate group of TMCM onto arabinogalactan, TMCM itself or proteins such as PorH [32, 33, 46, 47]. C. glutamicum possesses six corynomycolyl transferase genes, cop1, cmt1, cmt2, cmt3, cmt4 and cmt5 [32], and Cop1, Cmt1 and Cmt2 catalyze TDCM synthesis from TMCM [32, 46]. Cop1 is also reported to transfer corynomycolate from TMCM to arabinogalactan in the cell wall in $C$. glutamicum CGL2005 [33]. Interestingly, sigD overexpression induced the expression of four out of six corynomycolyl transferase genes at the same time, and enhanced the secretion of Cmt1 and $\mathrm{Cmt} 2$ to the supernatant. PorH forms the major cell wall channel penetrating the mycomembrane together with the other protein PorA [48], and corynomycolation of PorH and PorA catalyzed by Cmt1 was shown to be necessary for the pore forming activity $[47,49]$. Furthermore, ElrF was identified as the envelope lipids regulation factor which regulates lipid composition 


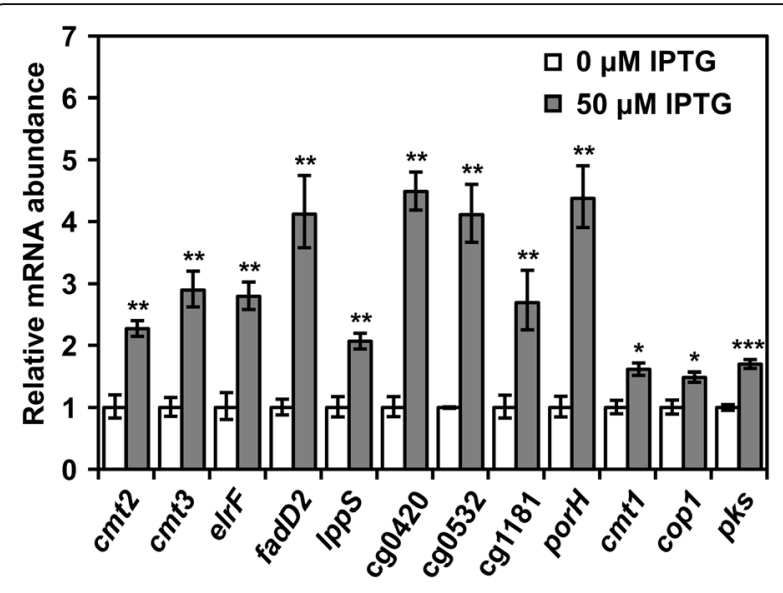

Fig. 4 Relative $m R N A$ abundance of genes upregulated during sigD overexpression. Relative abundance of mRNA of each gene was quantified by real-time PCR. The white and gray columns show the abundance in WT(pWWEx1-sigD) without IPTG (0 $\mu$ M IPTG), and in WT(pWWEx1-sigD) with $50 \mu \mathrm{M}$ of IPTG (50 $\mu$ M IPTG), respectively. Error bars represent standard deviations calculated from biological triplicates. The $p$-value of mRNA abundance was calculated by Student's t-test (two-tail, unpaired) between $0 \mu \mathrm{M}$ and $50 \mu \mathrm{M}$ of IPTG, and is shown by *, ** and *** for $<0.05,<0.01$ and $<0.001$, respectively

of corynomycolic acids and phospholipids in cell envelope [50]. In this study, increased content of TDCM in the crude lipid extract indicated that $\operatorname{sig} D$ overexpression influences not only the transcription of those genes but also the metabolic flux toward mycomembrane synthesis (Fig. 6). These results indicate that SigD controls the integrity of the cell envelope, especially of the mycomembrane in C. glutamicum.
SigD of C. glutamicum is classified as ECF40 type sigma factor by the ECFfinder program [3], as is SigD of M. tuberculosis. SigD in M. tuberculosis was shown to be essential for virulence, and inactivation of sigD decreased expression of some mycolyl transferase genes as well as other genes related to lipid metabolism and cell wall processes [51, 52]. For example, Calamita et al. reported that the expression of $f b p A$ encoding antigen $85 \mathrm{~A}$, which is a homolog for corynomycolyl transferase, decreased in the $\operatorname{sig} D$ deletion strain [52]. Raman et al. showed that the expression of $f b p C$ encoding antigen $85 \mathrm{C}$, which is also a homolog for corynomycolyl transferase, decreased twofold in the $\operatorname{sig} D$ deletion strain [51]. Even though a different organism has a different regulatory architecture, $C$. glutamicum and $M$. tuberculosis may share the similar regulatory network by SigD.

SigD gene overexpression in C. glutamicum led to excretion of carbohydrate-containing compounds and cell aggregation. In C. glutamicum CGL2005, various types of polysaccharides were detected extracellularly [34, 53]. In addition, C. glutamicum CCTCC M201005 was found in soil as a producer of a bioflocculant consisting of galacturonic acid as the main structural unit [54]. In M. tuberculosis and M. smegmatis, polysaccharides containing arabinose are suggested to be involved in the aggregation of cells via interaction with Antigen 85s, which are homologs for corynomycolyl transferases [55]. Furthermore, arabinose was shown to promote the cell aggregation of M. smegmatis [56]. Therefore, excretion of polysaccharides or carbohydrate-containing compounds caused by $\operatorname{sig} D$ overexpression may induce cell aggregation also in C. glutamicum.

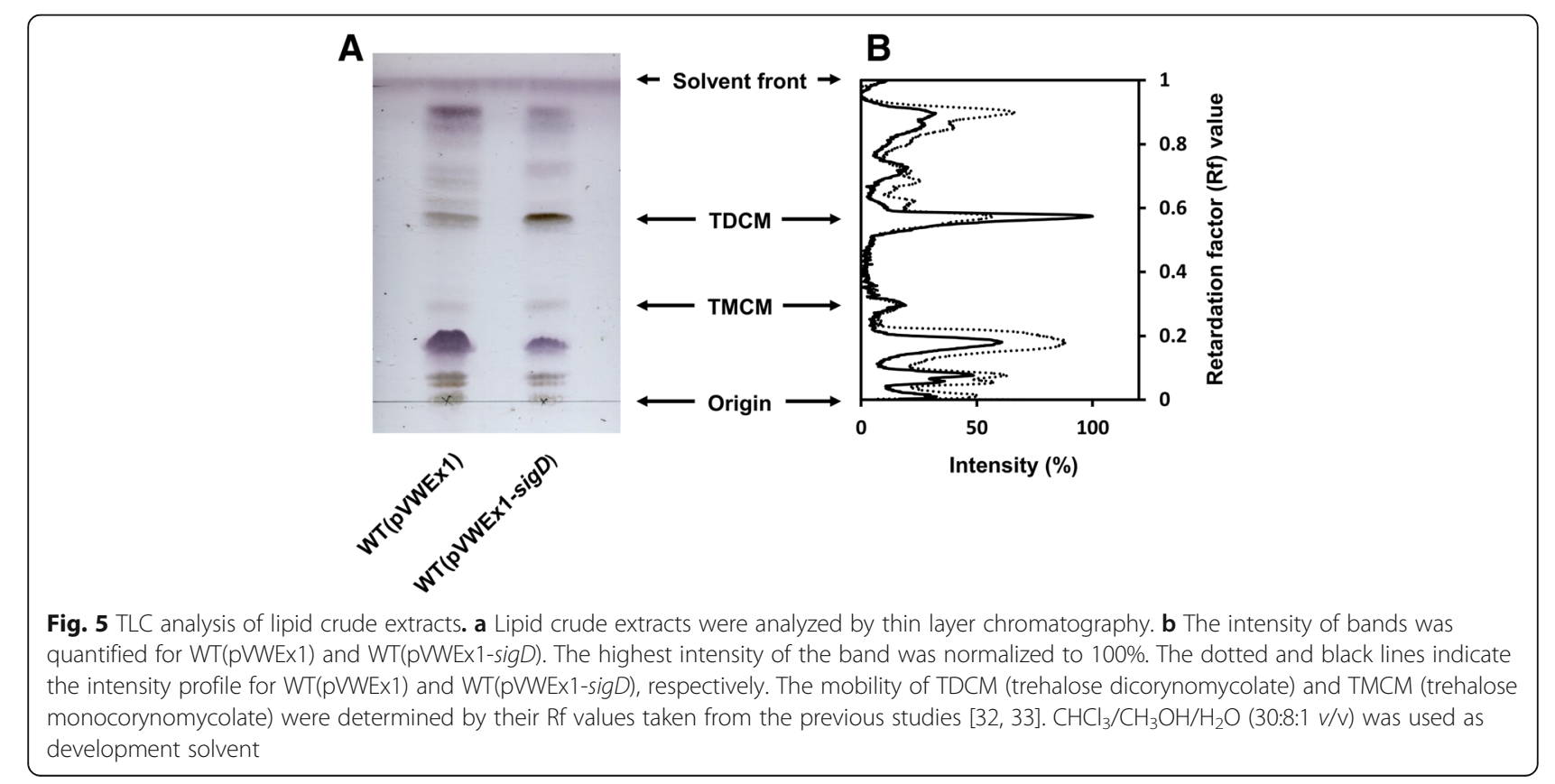




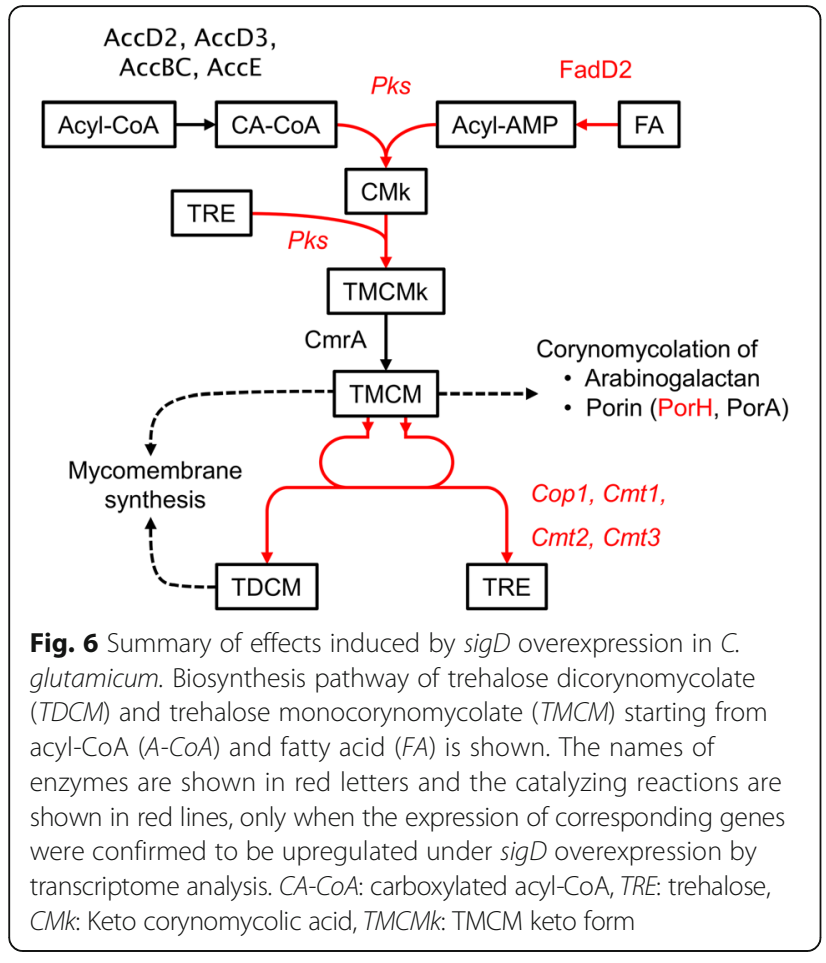

Sigma factors regulate transcription in a global manner and their effects are pleiotropic. In this study, the link between $\operatorname{sig} D$ overexpression and the regulation of cell wall integrity was confirmed, however, other effects remain to be elucidated, if exist. Furthermore, overexpression of sigD turns on the cascade regulation which includes direct and indirect outcomes inside the cells. Based on our results, the physiological role of C. glutamicum SigD in cell wall integrity seems apparent, however, the elucidation of the SigD regulon control and definition of the class of SigD-dependent promoters will require further molecular studies.

The non-pathogenic C. glutamicum serves as a good model organism for understanding the cell wall biosynthesis and resistance to antibiotics in Corynebacterineae, which include human pathogenic bacteria such as $M$. tuberculosis and C. diphtheriae [42]. Therefore, the findings described in this work can be helpful to understand the cell wall biosynthesis of Corynebacterineae. Furthermore, TDCM was shown to induce priming and activation of macrophages in vivo and in vitro in a similar manner as TDM from M. tuberculosis [57]. Chemical synthesis of TDM is not easy because it requires multiple steps, and those compounds are extracted from organisms. Considering the common biotechnological use of $C$. glutamicum, the production of TDCM with C. glutamicum seems to be an attractive idea. On the other hand, a corynomycolate-less strain is known to excrete more Lglutamate and L-lysine [58] as well as to take up glycerol and acetate more efficiently [33]. Reorganization of the mycomembrane by controlling sigD expression can be therefore helpful to understand the permeability barrier of C. glutamicum cells, and construct strains with higher or lower permeability barriers.

\section{Conclusion}

In this work, the functions of C. glutamicum sigma factor SigD were studied by overexpression or disruption of $\operatorname{sig} D$ gene. Overexpression of sigD led to the several physiological changes such as slower growth, cell aggregation, less foaming of the culture and increased turbidity of the supernatant. The real-time PCR analysis confirmed that overexpression of $\operatorname{sig} D$ induced the expression of several genes related to maintenance of cell envelop integrity and mycomembrane biosynthesis. Furthermore, overexpression of $\operatorname{sig} D$ increased the content of trehalose dicorynomycolate in the lipid extract.

\section{Additional files}

Additional file 1: Table S1. Bacterial strains, plasmids and oligonucleotides used in this work. Bacterial strains, plasmids and oligonucleotides used in this work are listed. (DOCX $17 \mathrm{~kb}$ )

Additional file 2: Table S2. RNA-seq analysis of genes differentially transcribed upon sigD overexpression or sigD disruption. The name of genes which expression levels increased under sigD overexpression (M-value > 1.0) are listed. (DOCX $18 \mathrm{~kb})$

\section{Abbreviations}

IPTG: Isopropyl $\beta$-D-1-thiogalactopyranoside; TDCM: Trehalose dicorynomycolate; TDM: Trehalose dimycolate; TMCM: Trehalose monocorynomycolate

\section{Acknowledgements}

Not applicable.

\section{Funding}

HT acknowledges support by DAAD (Deutscher Akademischer Austauschdienst) for providing a full PhD fellowship and MP acknowledges support by grant 17-06991S from Czech Science Foundation. Those foundations had no role in the design of the study in collection, analysis, and interpretation of data and in writing the manuscript.

\section{Availability of data and materials}

The datasets generated during the current study are available in the ArrayExpress database repository (E-MTAB-5411: http://www.ebi.ac.uk/ arrayexpress/).

\section{Authors' contributions}

$H T, T B, T P, K N, M P, J K$ and VFW designed the study. HT, TB, TP performed the experiments and analyzed the data. HT wrote the manuscript. TB, TP, KN, MP and JK reviewed the manuscript. VFW finalized the manuscript. All authors read and approved the final manuscript.

\section{Author's information}

HT currently belongs to Synthetic Bioengineering lab, Dept.of Biotechnology, Graduate School of Engineering, Osaka University (Yamadaoka 2-1, Suita,

Osaka, 565-0871, Japan).

Ethics approval and consent to participate

Not applicable.

Consent for publication

Not applicable. 


\section{Competing interests}

The authors declare that they have no competing interests.

\section{Publisher's Note}

Springer Nature remains neutral with regard to jurisdictional claims in published maps and institutional affiliations.

\section{Author details}

${ }^{1}$ Genetics of Prokaryotes, Faculty of Biology, Bielefeld University, Bielefeld, Germany. ${ }^{2}$ Center for Biotechnology, Bielefeld University, Bielefeld, Germany. ${ }^{3}$ Proteome and Metabolome Research, Faculty of Biology, Bielefeld University, Bielefeld, Germany. ${ }^{4}$ Institute of Microbiology, Academy of Sciences of the Czech Republic, Prague, Czech Republic.

\section{Received: 3 May 2017 Accepted: 4 July 2017}

\section{Published online: 12 July 2017}

\section{References}

1. Feklístov A, Sharon BD, Darst SA, Gross CA. Bacterial sigma factors: a historical, structural, and genomic perspective. Annu Rev Microbiol. 2014;68:357-76

2. Rodrigue S, Provvedi R, Jacques P-E, Gaudreau L, Manganelli R. The sigma factors of Mycobacterium tuberculosis. FEMS Microbiol Rev. 2006;30:926-41.

3. Staroń A, Sofia HJ, Dietrich S, Ulrich LE, Liesegang H, Mascher T. The third pillar of bacterial signal transduction: classification of the extracytoplasmic function (ECF) $\sigma$ factor protein family. Mol Microbiol. 2009;74:557-81.

4. Nicolas P, Mäder U, Dervyn E, Rochat T, Leduc A, Pigeonneau N. Conditiondependent transcriptome reveals high-level regulatory architecture in Bacillus subtilis. Science. 2012:335:1103-6.

5. Cho B-K, Kim D, Knight EM, Zengler K, Palsson BO. Genome-scale reconstruction of the sigma factor network in Escherichia coli: topology and functional states. BMC Biol. 2014;12:4.

6. Österberg S, Peso-Santos T de., and Shingler V. Regulation of alternative sigma factor use. Annu Rev Microbiol 2011;65:37-55.

7. Kinoshita S, Udaka S, Shimono M. Studies on the amino acid fermentation. J Gen Appl Microbiol. 1957;3:193-205.

8. Eggeling L, Bott M. Handbook of Corynebacterium glutamicum. FL, USA: CRC Press; 2005

9. Kalinowski J, Bathe B, Bartels D, Bischoff N, Bott M, Burkovski A. The complete Corynebacterium glutamicum ATCC 13032 genome sequence and its impact on the production of L-aspartate-derived amino acids and vitamins. J Biotechnol. 2003:104:5-25.

10. Ikeda M, Nakagawa S. The Corynebacterium glutamicum genome: features and impacts on biotechnological processes. Appl Microbiol Biotechnol. 2003;62:99-109.

11. Pátek $M$, Nešvera J. Sigma factors and promoters in Corynebacterium glutamicum. J Biotechnol. 2011;154:101-13.

12. Barksdale L. Corynebacterium diphtheriae and its relatives. Bacteriol Rev. 1970;34:378-422

13. Lanéelle M-A, Tropis M, Daffé M. Current knowledge on mycolic acids in Corynebacterium glutamicum and their relevance for biotechnological processes. Appl Microbiol Biotechnol. 2013;97:9923-30.

14. Peters-Wendisch PG, Schiel B, Wendisch VF, Katsoulidis E, Möckel B, Sahm H. Pyruvate carboxylase is a major bottleneck for glutamate and lysine production by Corynebacterium glutamicum. J Mol Microbiol Biotechnol. 2001;3:295-300

15. Schäfer A, Tauch A, Jäger W, Kalinowski J, Thierbach G, Pühler A. Small mobilizable multi-purpose cloning vectors derived from the Escherichia coli plasmids pK18 and pK19: selection of defined deletions in the chromosome of Corynebacterium glutamicum. Gene. 1994;145:69-73.

16. Sambrook J. Molecular cloning: A laboratory manual. third ed. Cold Spring Harbor: Cold Spring Harbor Laboratory Press; 2001

17. Gibson DG, Young L, Chuang R-Y, Venter JC, Hutchison CA, Smith HO Enzymatic assembly of DNA molecules up to several hundred kilobases. Nat Methods. 2009;6:343-5.

18. Nováková J, Izsáková A, Grivalský T, Ottmann C, Farkašovský M. Improved method for high-efficiency electrotransformation of Escherichia coli with the large BAC plasmids. Folia Microbiol (Praha). 2014;59:53-61.

19. van der Rest ME, Lange C, Molenaar D. A heat shock following electroporation induces highly efficient transformation of Corynebacterium glutamicum with xenogeneic plasmid DNA. Appl Microbiol Biotechnol. 1999;52:541-5.
20. Shevchenko A, Tomas H, Havlis J, Olsen JV, Mann M. In-gel digestion for mass spectrometric characterization of proteins and proteomes. Nat Protoc. 2006;1:2856-60.

21. Musa YR, Bäsell K, Schatschneider S, Vorhölter F-J, Becher D, Niehaus K. Dynamic protein phosphorylation during the growth of Xanthomonas campestris pv. campestris B100 revealed by a gel-based proteomics approach. J Biotechnol. 2013;167:111-22.

22. Dallies N, François J, Paquet V. A new method for quantitative determination of polysaccharides in the yeast cell wall. Application to the cell wall defective mutants of Saccharomyces cerevisiae. Yeast. 1998;14:1297-306.

23. DuBois M, Gilles KA, Hamilton JK, Rebers PA, Smith F. Colorimetric method for determination of sugars and related substances. Anal Chem. 1956;28:350-6.

24. Masuko T, Minami A, Iwasaki N, Majima T, Nishimura S-I, Lee YC. Carbohydrate analysis by a phenol-sulfuric acid method in microplate format. Anal Biochem. 2005;339:69-72.

25. Hüser AT, Becker A, Brune I, Dondrup M, Kalinowski J, Plassmeier J. Development of a Corynebacterium glutamicum DNA microarray and validation by genome-wide expression profiling during growth with propionate as carbon source. J Biotechnol. 2003;106:269-86.

26. Busche T, Winkler A, Wedderhoff I, Rückert C, Kalinowski J, Ortiz de Orué Lucana D. Deciphering the Transcriptional Response Mediated by the Redox-Sensing System HbpS-SenS-SenR from Streptomycetes. PLoS One. 2016;11:e0159873.

27. Blom J, Jakobi T, Doppmeier D, Jaenicke S, Kalinowski J, Stoye J, et al. Exact and complete short-read alignment to microbial genomes using Graphics Processing Unit programming. Bioinforma Oxf Engl. 2011;27:1351-8.

28. Pfeifer-Sancar K, Mentz A, Rückert C, Kalinowski J. Comprehensive analysis of the Corynebacterium glutamicum transcriptome using an improved RNAseg technique. BMC Genomics. 2013;14:888.

29. Hilker R, Stadermann KB, Schwengers O, Anisiforov E, Jaenicke S, Weisshaar B, et al. ReadXplorer 2-detailed read mapping analysis and visualization from one single source. Bioinforma Oxf Engl. 2016:32:3702-8.

30. Anders $S$, Huber W. Differential expression analysis for sequence count data. Genome Biol. 2010;11:106.

31. Busche T, Silar R, Pičmanová M, Pátek M, Kalinowski J. Transcriptional regulation of the operon encoding stress-responsive ECF sigma factor $\mathrm{SigH}$ and its anti-sigma factor RshA, and control of its regulatory network in Corynebacterium glutamicum. BMC Genomics. 2012;13:445.

32. Brand S, Niehaus K, Pühler A, Kalinowski J. Identification and functional analysis of six mycolyltransferase genes of Corynebacterium glutamicum ATCC 13032: the genes cop 1, cmt1, and cmt2 can replace each other in the synthesis of trehalose dicorynomycolate, a component of the mycolic acid layer of the cell envelope. Arch Microbiol. 2003;180:33-44.

33. Puech V, Bayan N, Salim K, Leblon G, Daffé M. Characterization of the in vivo acceptors of the mycoloyl residues transferred by the corynebacterial PS1 and the related mycobacterial antigens 85. Mol Microbiol. 2000;35:1026-41.

34. Puech V. Chami M, Lemassu A, Lanéelle M-A, Schiffler B, Gounon P. Structure of the cell envelope of corynebacteria: importance of the noncovalently bound lipids in the formation of the cell wall permeability barrier and fracture plane. Microbiology. 2001;147:1365-82.

35. Nielsen SS. Food Analysis Laboratory Manual. Springer US: NY, USA; 2010

36. Ikeda M, Baba M, Tsukumoto N, Komatsu T, Mitsuhashi S, Takeno S. Elucidation of genes relevant to the microaerobic growth of Corynebacterium glutamicum. Biosci Biotechnol Biochem. 2009;73:2806-8.

37. Taniguchi $H$, Wendisch VF. Exploring the role of sigma factor gene expression on production by Corynebacterium glutamicum: sigma factor $\mathrm{H}$ and FMN as example. Front Microbiol. 2015:6:740.

38. Taniguchi H, Henke NA, Heider SAE, Wendisch VF. Overexpression of the primary sigma factor gene sigA improved carotenoid production by Corynebacterium glutamicum: Application to production of $\beta$-carotene and the non-native linear C50 carotenoid bisanhydrobacterioruberin. Metab Eng Commun. 2017:4:1-11.

39. Gande R, Dover LG, Krumbach K, Besra GS, Sahm H, Oikawa T. The two carboxylases of Corynebacterium glutamicum essential for fatty acid and mycolic acid synthesis. J Bacteriol. 2007;189:5257-64.

40. Portevin D, de Sousa-D'Auria C, Montrozier H, Houssin C, Stella A, Lanéelle M-A. The acyl-AMP ligase FadD32 and AccD4-containing acyl-CoA carboxylase are required for the synthesis of mycolic acids and essential for mycobacterial growth: identification of the carboxylation product and determination of the acyl-CoA carboxylase components. J Biol Chem. 2005;280:8862-74. 
41. Gavalda S, Bardou F, Laval F, Bon C, Malaga W, Chalut C. The polyketide synthase Pks13 catalyzes a novel mechanism of lipid transfer in mycobacteria. Chem Biol. 2014;21:1660-9.

42. Portevin D, De Sousa-D'Auria C, Houssin C, Grimaldi C, Chami M, Daffé M, et al. A polyketide synthase catalyzes the last condensation step of mycolic acid biosynthesis in mycobacteria and related organisms. Proc Natl Acad Sci U S A. 2004;101:314-9.

43. Lea-Smith DJ, Pyke JS, Tull D, McConville MJ, Coppel RL, Crellin PK. The reductase that catalyzes mycolic motif synthesis is required for efficient attachment of mycolic acids to arabinogalactan. J Biol Chem. 2007;282:11000-8

44. Varela C, Rittmann D, Singh A, Krumbach K, Bhatt K, Eggeling L. MmpL genes are associated with mycolic acid metabolism in mycobacteria and corynebacteria. Chem Biol. 2012;19:498-506.

45. Yamaryo-Botte Y, Rainczuk AK, Lea-Smith DJ, Brammananth R, van der Peet $\mathrm{PL}$, Meikle P. Acetylation of trehalose mycolates is required for efficient mmpl-mediated membrane transport in Corynebacterineae. ACS Chem Biol. 2015;10:734-46

46. De Sousa-D'Auria C, Kacem R, Puech V, Tropis M, Leblon G, Houssin C. New insights into the biogenesis of the cell envelope of corynebacteria: identification and functional characterization of five new mycoloyltransferase genes in Corynebacterium glutamicum. FEMS Microbiol Lett. 2003;224:35-44.

47. Huc E, de Sousa-D'Auria C, de la Sierra-Gallay IL, Salmeron C, van Tilbeurgh H, Bayan N. Identification of a mycoloyl transferase selectively involved in Oacylation of polypeptides in Corynebacteriales. J Bacteriol. 2013;195:4121-8.

48. Burkovski A. Cell envelope of corynebacteria: structure and influence on pathogenicity. ISRN Microbiol. 2013;2013:935736.

49. Barth E, Barceló MA, Kläckta C, Benz R. Reconstitution experiments and gene deletions reveal the existence of two-component major cell wall channels in the genus Corynebacterium. J Bacteriol. 2010;192:786-800.

50. Meniche X, Labarre C, de Sousa-d'Auria C, Huc E, Laval F, Tropis M, et al. Identification of a stress-induced factor of Corynebacterineae that is involved in the regulation of the outer membrane lipid composition. J Bacteriol. 2009;191:7323-32.

51. Raman S, Hazra R, Dascher CC, Husson RN. Transcription regulation by the Mycobacterium tuberculosis alternative sigma factor SigD and its role in virulence. J Bacteriol. 2004:186:6605-16.

52. Calamita H, Ko C, Tyagi S, Yoshimatsu T, Morrison NE, Bishai WR. The Mycobacterium tuberculosis SigD sigma factor controls the expression of ribosome-associated gene products in stationary phase and is required for full virulence. Cell Microbiol. 2004;7:233-44.

53. Kacem R, De Sousa-D'Auria C, Tropis M, Chami M, Gounon P, Leblon G. Importance of mycoloyltransferases on the physiology of Corynebacterium glutamicum. Microbiol Read Engl. 2004;150:73-84.

54. He N, Li Y, Chen J, Lun S-Y. Identification of a novel bioflocculant from a newly isolated Corynebacterium glutamicum. Biochem Eng J. 2002;11:137-48,

55. Anton $V$, Rougé $P$, Daffé $M$. Identification of the sugars involved in mycobacterial cell aggregation. FEMS Microbiol Lett. 1996;144:167-70.

56. Jayawardana KW, Wijesundera SA, Yan M. Aggregation-based detection of $M$. smegmatis using D-arabinose-functionalized fluorescent silica nanoparticles. Chem Commun. 2015;51:15964-6.

57. Chami M, Andréau K, Lemassu A, Petit J-F, Houssin C, Puech V. Priming and activation of mouse macrophages by trehalose 6,6-dicorynomycolate vesicles from Corynebacterium glutamicum. FEMS Immunol Med Microbiol. 2002;32:141-7.

58. Gebhardt H, Meniche X, Tropis M, Krämer R, Daffé M, Morbach S. The key role of the mycolic acid content in the functionality of the cell wall permeability barrier in Corynebacterineae. Microbiology. 2007;153:1424-34.

\section{Submit your next manuscript to BioMed Central and we will help you at every step:}

- We accept pre-submission inquiries

- Our selector tool helps you to find the most relevant journal

- We provide round the clock customer support

- Convenient online submission

- Thorough peer review

- Inclusion in PubMed and all major indexing services

- Maximum visibility for your research

Submit your manuscript at www.biomedcentral.com/submit
Biomed Central 